\title{
Optimal Control of the Separation Process with Flow Restrictions
}

\author{
Nikolai D. Demidenko ${ }^{a}$, \\ Lyudmila V. Kulagina ${ }^{* b}$ and Aleksandr G. Nikiforov ${ }^{\mathrm{c}}$ \\ ${ }^{a}$ Krasnoyarsk Branch - \\ Nauka Specialized Design Technology Office \\ Institute of Computational Technologies SB RAS \\ 53 Mira, Krasnoyarsk, 660049, Russia \\ ${ }^{b}$ Siberian Federal University \\ 79 Svobodny, Krasnoyarsk, 660041, Russia \\ 'Smolensk State Agricultural Academy \\ 10 Bolshaya Sovetskaya Str., Smolensk, 214000, Russia
}

Received 08.02.2019, received in revised form 10.02.2019, accepted 12.02.2019

Here we formulate the problem of optimal process control with distributed parameters, taking into account the constraints on the control and associated flows. The necessary optimality conditions are obtained. The analysis of the stationarity conditions is carried out and the method for constructing the domain of admissible controls is proposed. The developed optimization method is applied in the automation of industrial distillation plants for the sulfuric acid alkylation of isobutane with butylenes, ortho-xylene production, etc.

Keywords: optimal control, processes with distributed parameters, rectification, mathematical model.

Citation: Demidenko N.D., Kulagina L.V., Nikiforov A.G. Optimal control of the separation process with flow restrictions, J. Sib. Fed. Univ. Eng. technol., 2019, 12(2), 159-170. DOI: 10.17516/1999-494X-0125.

(c) Siberian Federal University. All rights reserved

This work is licensed under a Creative Commons Attribution-NonCommercial 4.0 International License (CC BY-NC 4.0).

* Corresponding author E-mail address: klvation@gmail.com 


\title{
Оптимальное управление процессом разделения \\ с ограничениями на потоки
}

\author{
Н.Д. Демиденко ${ }^{a}$, Л.В. Кулагина \\ ${ }^{a}$ Красноярский филиал-СКТБ «Наука» \\ Института вычислительных технологий СО РАН \\ Россия, 660049, Красноярск, пр. Мира, 53 \\ ${ }^{6}$ Сибирский федеральный университет \\ Россия, 660041, Красноярск, пр. Свободный, 79 \\ ${ }^{8}$ Смоленская государственная сельскохозяйственная академия \\ Россия, 214000, Смоленск, ул. Большая Советская, 10
}

Здесь формулируется задача оптимального управления процессом с распределенными параметрами, при этом учитываются ограничения на управляющие и связанные с ними потоки. Получены необходимые условия оптимальности. Проведен анализ условий стационарности и предложена методика построения области допустимых управлений. Разработанныцй метод оптимизации применен при автоматизации промышленных ректификационных установок сернокислотного алкилирования изобутана бутиленами, получения ортоксилола и др.

Ключевые слова: оптимальное управление, процессы с распределенными параметрами, ректификация, математическая модель.

\section{Введение}

В настоящее время мощное развитие получили теория и практика систем с распределенными параметрами [1-9]. Это обусловлено, с одной стороны, наличием современных наукоемких технологий в металлургии, нефтепереработке и нефтехимии, энергетике и других отраслях промышленности, с другой - разработанным перспективным математическим методом анализа гидротермодинамических процессов и систем управления с целью проектирования высокоэффективных производств [10-24]. Произошло весьма продуктивное соединение современного математического аппарата с нуждами практических проблем в промышленности и современными вычислительными технологиями. Важным обстоятельством выступает тот факт, что основу большинства непрерывных промышленных производств составляют процессы тепломассообмена в условиях сложных гидродинамических явлений, например в условиях суперкавитации $[11,13,23,24]$. Однако совместное рассмотрение и изучение вышеназванных процессов довольно сложная задача, и в последнее время в основном изучают с различных точек зрения теоретические и прикладные задачи раздельно, мало учитывая влияние одних процессов на другие.

Вычислительные технологии объединяют новейшие достижения математических методов с нуждами производства, несмотря на то, что они развиваются самостоятельно, но тем не менее влияют друг на друга. И только в вычислительных технологиях достигается совершенство математических абстракций и реального производства, решаются различные системы уравнений в частных производных в области постановки краевых задач, аналитических и численных методов, вопросы качественного исследования краевых задач и задач оптимального управления. Результатом этого эволюционного процесса могут являться разработанные науч- 
но обоснованные методы расчета основных технологических и конструктивных параметров (геометрических, гидротермодинамических и кавитационных) при проектировании надежных и высокопроизводительных технологических аппаратов в различных отраслях промышленности, в которых происходят процессы тепломассообмена и гидродинамики взаимодействующих потоков. Важными остаются вопросы степени адекватности математического описания этих процессов реальным режимам технологических объектов. Для различных условий адекватность может быть разной. Необходимо, чтобы точность математического описания соответствовала требованиям конкретного производства.

Значимым элементом вычислительных технологий являются системы с распределенными параметрами, которые функционируют в условиях управления, в том числе оптимального. Распределенность параметров процесса требует особых подходов при синтезе систем контроля и управления. В частности, для таких систем естественным образом необходимо применять распределенный контроль и распределенное управление [1-3, 10, 11].

В статье рассматривается задача оптимального управления процессами с рециркуляцией взаимодействующих потоков в ректификационных колоннах. Рециркуляция приводит к тому, что одни и те же управления как граничные, так и «объемные». Поэтому для вывода необходимых условий оптимальности здесь непосредственно применяется метод вариаций; управления предполагаются кусочно-непрерывными, а соответствующие им решения - непрерывными и кусочно-гладкими $[1,2,11]$.

\section{Постановка задачи}

Рассмотрим следующую модель управляемого процесса:

$$
\begin{aligned}
& \frac{\partial\left(H_{x} x\right)}{\partial t}-\frac{\partial(L x)}{\partial l}=k_{y}\left(y-y^{*}(x)\right)+F(t) \Phi_{x}(l) x_{F}, \\
& \frac{\partial\left(H_{y} y\right)}{\partial t}-\frac{\partial(V y)}{\partial l}=k_{y}\left(y^{*}(x)-y\right), \quad 0<t<T, \quad 0<l<1,
\end{aligned}
$$

краевые условия: при $l=0$ :

$$
\begin{aligned}
& \frac{d\left(H_{x_{k}} x_{k}\right)}{d t}=L(0, t) x(0, t)-V(0, t) y(0, t)-W x_{k}(t), \\
& y(0, t)=a\left[y_{k}^{*}\left(x_{k}\right)-x_{k}(t)\right]+x_{k}(t),
\end{aligned}
$$

при $l=1$ :

$$
\begin{aligned}
& \frac{d\left(H_{x_{d}} x_{d}\right)}{d t}=V_{d}(t) y_{d}-\left(L_{d}(t)+D(t)\right) x_{d}(t), \\
& V_{d} y_{d}(t)-V(1, t) y(1, t)=L_{d} x_{d}(t)-L(1, t) x(1, t),
\end{aligned}
$$

где $x(l, t), y(l, t)$ - концентрации целевого продукта в жидкой и паровой фазах; $L(l, t), V(l, t)-$ жидкий и паровой поток; $F$ - поток сырья; $\mathrm{y}^{*}(x)$ - равновесная концентрация в паровой фазе; $H x, H y$ - удерживающие способности в жидкой и паровой фазах; $x_{F}$ - концентрация целевого продукта в сырье; $D(t), W(t)$ - отбор целевого продукта вверху и внизу колонны; $k, d$ относятся к параметрам в кубе и дефлегматоре.

$$
-161-
$$


Начальные условия:

$$
\begin{aligned}
& x(l, 0)=x_{0}(l), \quad y(l, 0)=y_{0}(l), \quad 0<l<1, \\
& x_{d}(0)=x_{d 0}, \quad x_{k}(0)=x_{k 0}, \quad 0 \leq a, \quad E_{d} \leq 1 .
\end{aligned}
$$

$F(t)$ - поток в жидкой фазе, подводимый в колонну на промежутке $l_{0}-\sigma \leq l \leq l_{0}+\sigma ; \Phi_{x}(l)$ характеризует распределение потока по этому промежутку. Функция $\Phi_{x}$ может иметь, например, вид

$$
\Phi_{x}(l)=\left\{\begin{array}{lll}
e^{-\frac{\sigma^{2}}{\sigma^{2}-\left(l-l_{0}\right)^{2}}} & \text { при } & \left|l-l_{0}\right|<\sigma \\
0 & \text { при } & \left|l-l_{0}\right| \geq \sigma
\end{array}\right.
$$

или

$$
\Phi_{x}(l)=\left\{\begin{array}{lll}
\frac{1}{k} \cos ^{2 m}\left[n\left(l-l_{0}\right)\right] & \text { при } & \left|l-l_{0}\right|<\frac{\pi}{2 n}=\sigma \\
0 & \text { при } & \left|l-l_{0}\right| \geq \sigma
\end{array} .\right.
$$

Во втором случае $m$ выбирается достаточно большим для обеспечения нужной гладкости $\Phi_{x}(l)$; в обоих случаях $k$ выбирается так, чтобы

$$
\int_{l_{0}-\sigma}^{l_{0}+\sigma} \Phi_{x}(l) d l=1 .
$$

Величины потоков при этом удовлетворяют условиям:

$$
\begin{array}{ll}
W(t)+D(t)=F(t), \quad D(t)+L_{d}(t)=V(l, t), \\
W(t)+V(0, t)=L(0, t), & V_{d}(t)=V(l, t) .
\end{array}
$$

Предполагается, что удерживающие способности $H_{x}, H_{y}$ постоянны, $V$ не зависит от $l$, а $L(l, t)$ имеет вид

$$
L(l, t)= \begin{cases}L(l, t) & \text { npu } l_{0}+\sigma \leq l \leq 1 \\ L(l, t)+F(t) \int_{l_{0}-\sigma}^{l_{0}+\sigma} \Phi_{x}(l) d l & \text { npu } l_{0}-\sigma \leq l \leq l_{0}+\sigma, \\ L(l, t)+F(t) & \text { nрu } 0 \leq l \leq l_{0}-\sigma\end{cases}
$$

что короче будем записывать таким образом:

$$
L(l, t)=L(1, t)+L^{*}(l, t)=L(t)+L^{*}(l, t) .
$$

Такой вид $L$ соответствует предположению, что изменение потока в жидкой фазе по высоте колонны происходит только за счет вводимого потока $F(t)$. При этом условия (5) дают:

$$
\begin{array}{lll}
W(t)+D(t)=F(t), & D(t)+L_{d}(t)=V(t), \\
W(t)+V(t)=L(t)+F(t), & V_{d}(t)=V(t) .
\end{array}
$$

Из этих равенств следует, что $L_{d}(t)=L(t)=L(1, t)$.

Анализ условий (5) показывает, что только два из четырех потоков $W, D, L, V$ являются независимыми. Выбор тех или иных двух независимых потоков в качестве управлений опре- 
деляет соответствующие задачи. Эти задачи, как задачи оптимального управления в классе кусочно-непрерывных управлений с критерием качества

$$
F=\int_{0}^{T} \int_{0}^{1}\left(y(l, t)-\theta^{*}(l, t)\right)^{2} d l d t,
$$

где $\theta^{*}$ - заданная функция.

\section{Задача оптимального управления \\ с управляющими потоками $L, W$}

Здесь рассматривается задача оптимального управления, в которой управляющими параметрами являются потоки $L=L(t)$ и $W=W(t)$. Потоки $V, D, L_{d}, V_{d}$ исключаются с помощью условий (5). Управления $L, W$ выбираются в классе кусочно-непрерывных функций, принимающих значения в промежутках

$$
L_{\min } \leq L(t) \leq L_{\max }, \quad W_{\min } \leq W(t) \leq W_{\max }
$$

поток $F$ считается заданным. Переходя к нормальной форме дифференциальных уравнений, получаем следующую задачу:

$$
\begin{aligned}
& x_{t}^{\prime}=\frac{1}{H_{x}}\left[\left(L+L^{*}\right) \cdot \varsigma^{(1)}+\frac{\partial L}{\partial l} x+k_{y}\left(y-y^{*}\right) F \Phi_{x} x_{F}\right] \equiv X, \quad x_{l}^{\prime}=\varsigma^{(1)}, \\
& y_{t}^{\prime}=\frac{1}{H_{y}}\left[(W-L-F) \cdot \varsigma^{(2)}+k_{y}\left(y^{*}-y\right)\right] \equiv Y, \quad y_{l}^{\prime}=\varsigma^{(2)}, \quad 0<t<T, \quad 0<l<1,
\end{aligned}
$$

при краевых условиях:

$$
\begin{aligned}
& x^{\prime}{ }_{k t}=\frac{1}{H_{x_{k}}}\left[(L+F) x+(W-L-F) y-W x_{k}\right] \equiv X_{k}, \\
& y-a \cdot\left[y_{k}^{*}\left(x_{k}\right)-x_{k}\right]-x_{k}=0, \quad 0<t<T, \quad l=0 ; \\
& x_{d t}^{\prime}=\frac{1}{H_{x_{d}}}[L+F-W]\left(y_{d}-x_{d}\right) \equiv X_{d}, \\
& (L+F-W) \cdot\left(y_{d}-y\right)-L\left(x_{d}-x\right)=0, \\
& y_{d}-y-E_{d}\left(y_{d}^{*}\left(x_{d}\right)-y\right)=0, \quad 0<t<T, \quad l=1,
\end{aligned}
$$

при начальных условиях (4) и ограничениях на управления:

$$
\left(L-L_{\text {min }}\right) \cdot\left(L_{\max }-L\right)-u^{2}=0, \quad\left(W-W_{\min }\right) \cdot\left(W_{\max }-W\right)-z^{2}=0 .
$$

Здесь $u, z$ - вспомогательные управления.

Будем учитывать также ограничения на потоки $D$ и $V$ :

$$
\begin{aligned}
& D_{\min } \leq D(t) \leq D_{\max ,} \text {, где } \quad D=F-W, \\
& V_{\min } \leq V(t) \leq V_{\max }, \quad \text { где } \quad V=L+F-W .
\end{aligned}
$$

Из последних неравенств следует 


$$
\begin{aligned}
& W_{\min } \leq W(t) \leq W_{\max }, \quad \text { или } \quad W_{\min } \leq W(t) \leq W_{\max }, \\
& D_{\min } \leq F-W \leq D_{\max }, \quad F-D_{\max } \leq W \leq F-D_{\min }, \\
& V_{\text {min }} \leq L+F-W \leq V_{\max }, \quad L_{\text {min }}+F-V_{\max } \leq W \leq F+L_{\max }-V_{\min } .
\end{aligned}
$$

Итак,

$$
\begin{aligned}
& \widetilde{W}_{\min }=\max \left\{W_{\min }, F-D_{\max }, F+L_{\text {min }}-V_{\max }\right\} \leq \\
& \leq W \leq \min \left\{W_{\max }, F-D_{\min }, F+L_{\text {max }}-V_{\min }\right\}=\widetilde{W}_{\max }
\end{aligned}
$$

при условии, что любой элемент множества $\left\{W_{\min }, F-D_{\max }, F+L_{\min }-V_{\max }\right\}$ не превосходит любой элемент множества $\left\{W_{\max }, F-D_{\min }, F+L_{\max }-V_{\min }\right\}$. Это означает, что параметры задачи должны удовлетворять условиям

$$
V_{\min } \leq L_{\max }+D_{\max }, \quad V_{\max } \geq L_{\min }+D_{\min },
$$

а поток $F$ должен удовлетворять следующим соотношениям:

$$
\begin{aligned}
& F \geq W_{\min }+D_{\min }, \quad F \leq W_{\max }+D_{\max }, \\
& F \geq W_{\min }+V_{\min }-L_{\max }, \quad F \leq W_{\max }+V_{\max }-L_{\min } .
\end{aligned}
$$

Будем предполагать, что эти условия выполнены. Заметим, что необходимость выполнения первых четырех из условий (13)-(14) естественно вытекает из первых двух условий (5).

Аналогично можно получить соотношение

$$
L_{\min }(W)=\max \left\{L_{\min }, W-F+V_{\min }\right\} \leq L(t) \leq \min \left\{L_{\max }, W-F+V_{\max }\right\}=L_{\max }(W) .
$$

При этом границы изменения $W$ постоянны или определяются потоком $F$, а границы изменения $L$ зависят от управления $W$. Получаем следующую задачу: для процесса, описываемого уравнениями (4), (9)-(11), в множестве кусочно-непрерывных управлений $L, W$, удовлетворяющих условиям

$$
\left(W-\widetilde{W}_{\min }\right)\left(\widetilde{W}_{\max }-W\right)-z^{2}=0, \quad\left(L-L_{\min }(W)\right)\left(L_{\max }(W)-L\right)-u^{2}=0,
$$

найти такие, что соответствующее им решение задачи (9)-(11), (15), (16), (4) дает минимум интегралу (7).

\section{Необходимые условия оптимальности}

Для получения необходимых условий оптимальности рассматривается вспомогательный интеграл

$$
\begin{aligned}
& I=I_{1}+I_{2}=\iint_{\Omega} \widetilde{L} d l d t+\iint_{\partial \Omega} \widetilde{l} d t, \\
& \text { əde } \widetilde{L}=\left(y-\theta^{*}\right)^{2}+\xi^{(1)}\left(x_{t}^{\prime}-X\right)+\xi^{(2)}\left(x_{l}^{\prime}-\zeta^{(1)}\right)+\eta^{(1)}\left(y_{t}^{\prime}-Y_{t}\right)+\eta^{(2)}\left(y_{l}^{\prime}-\zeta^{(2)}\right), \\
& \tilde{l}=\lambda_{k}^{(1)}\left(x_{k t}^{\prime}-X_{k}\right)+\lambda_{k}^{(2)}\left(y-x_{k}-a \cdot\left(y_{k}^{*}\left(x_{k}\right)-x_{k}\right)\right)+\lambda_{d}^{(1)}\left(x_{d t}^{\prime}-X_{d}\right)+ \\
& +\lambda_{s}^{(2)}\left((L+F-W) \cdot\left(y_{d}-y\right)-L\left(x_{d}-x\right)\right)+\lambda_{s}^{(3)}\left(y_{d}-y-E_{d}\left(y_{d}^{*}-y\right)\right)+\gamma\left(\left(L-L_{\min }(W)\right) .\right. \\
& \left.\left(L_{\max }(W)-L\right)-u^{2}\right)+\varepsilon\left(\left(W-\widetilde{W}_{\min }\right) \cdot\left(\widetilde{W}_{\max }-W\right)-z^{2}\right), \\
& -164-
\end{aligned}
$$


где $\xi^{(1)}, \xi^{(2)}, \eta^{(1)}, \eta^{(2)}-$ функции, определенные на $\Omega=\{(l, t) \mid 0<l<1,0<t<T\} ; \lambda_{k}^{(i)}, \quad \lambda_{d}^{(i)}, \quad \gamma ; \varepsilon-$ функции, определенные на $[0, T]$ (их можно считать определенными на $\partial \Omega$ ), причем

$$
\begin{array}{lll}
\varepsilon=\lambda_{k}^{(i)}=0 & \text { на } & \partial \Omega /\{(l, t) \mid l=0\}, \\
\gamma=\lambda_{d}^{(i)}=0 & \text { на } & \partial \Omega /\{(l, t) \mid l=1\} .
\end{array}
$$

Пусть $L, W$ - оптимальные управления; $u, z$ - соответствующие им (согласно (16)) вспомогательные управления; $x, y, x_{k}, x_{d}, y_{d}$ - оптимальное решение задачи (9)-(11), (4), отвечающее этим управлениям; $\delta L, \delta W$ - вариации управлений $L, W ; \delta u, \delta z-$ вариации фиктивных управлений $u, z, \delta x, \delta y, \delta x_{k}, \delta x_{d}, \delta y_{d}$ - соответствующие вариации решений. Пользуясь аргументацией теории вариационного исчисления, получаем необходимые условия оптимальности управляющих функций $L$ и $W$ :

при $0<t<T, 0<l<1$

$$
\begin{aligned}
& \xi_{t}^{\prime}-\frac{L+L^{*}}{H_{x}} \xi_{l}^{\prime}=k_{y}\left(y^{*}\right)^{\prime}\left(\frac{\xi}{H_{x}}-\frac{\eta}{H_{y}}\right), \\
& \eta_{t}^{\prime}-\frac{L+F-W}{H_{y}} \eta_{l}^{\prime}=-k_{y}\left(\frac{\xi}{H_{x}}-\frac{\eta}{H_{y}}\right)+2\left(y-\theta^{*}\right) ;
\end{aligned}
$$

при $l=0,0<t<T$ :

$$
\begin{aligned}
& \frac{\xi}{H_{x}}-\frac{\lambda_{k}^{(1)}}{H_{x_{k}}}=0, \quad\left[\frac{\xi}{H_{y}}-\frac{\lambda_{k}^{(1)}}{H_{x_{k}}}\right] \cdot(L+F-W)-\lambda_{k}^{(2)}=0, \\
& \frac{d \lambda_{k}^{(1)}}{d t}=\frac{W}{H_{x_{k}}} \lambda_{k}^{(1)}+\lambda_{k}^{(2)}\left(-a\left(y_{k}^{*}\right)^{\prime}+a-1\right), \quad \lambda_{k}^{(1)}(T)=0 ;
\end{aligned}
$$

при $t-T, 0<l<1: \xi=0, \eta=0$;

при $l=1,0<t<T$ :

$$
\begin{aligned}
& \frac{d \lambda_{d}^{(1)}}{d t}=\frac{(L+F-W)}{H_{x_{d}}} \lambda_{d}^{(1)}-L \lambda_{d}^{(2)}, \quad \lambda_{d}^{(1)}(T)=0, \\
& {\left[\frac{\eta}{H_{y}}+\lambda_{d}^{(2)}\right] \cdot(L+F-W)+\left(1-E_{d}\right) \lambda_{d}^{(3)}=0,} \\
& {\left[\frac{\xi}{H_{x}}+\lambda_{d}^{(3)}\right] \cdot L=\lambda_{d}^{(2)} E_{d}\left(y_{d}^{*}\right)^{\prime}=0, \quad\left[\frac{\lambda_{d}^{(1)}}{H_{x_{d}}}+\lambda^{(2)}\right] \cdot(L+F-W)-\lambda_{d}^{(3)}=0 ;}
\end{aligned}
$$

при $0<t<T$ :

$$
\begin{aligned}
& \int_{0}^{1}\left(-\frac{\xi}{H_{x}} x_{l}^{\prime}+\frac{\eta}{H_{y}} y_{l}^{\prime}\right) d l-\frac{\lambda_{k}^{(1)}}{H_{x_{k}}}(x(0, t)-y(0, t))+\frac{\lambda_{d}^{(1)}}{H_{x_{d}}}\left(y_{d}-x_{d}\right)+ \\
& +\lambda_{d}^{(2)}\left(y(1, t)-y_{d}+x_{d}-x(1, t)\right)-\gamma\left(L_{\min }+L_{\max }-2 L\right)=0, \\
& \int_{0}^{1}-\frac{\eta}{H_{y}} y_{l}^{\prime} d l+\frac{\lambda_{k}^{(1)}}{H_{x_{k}}}\left(x_{k}-y(0, t)\right)-\frac{\lambda_{d}^{(1)}}{H_{x_{d}}}\left(y_{d}-x_{d}\right)-\lambda_{d}^{(2)}\left(y(1, t)-y_{d}\right)+ \\
& +\varepsilon\left(W_{\min }+W_{\max }-2 W\right)=0,
\end{aligned}
$$




$$
y u=0, \varepsilon z=0 .
$$

Так как уравнения (21), (22) определяют функции $\xi, \eta, \lambda_{k}^{(1)}, \lambda_{k}^{(2)}, \lambda_{d}^{(1)}, \lambda_{d}^{(2)}, \lambda_{d}^{(3)}$ полностью, то случаи, когда функции $\gamma, \varepsilon$ равны нулю, связаны с дополнительными жесткими условиями на параметры задачи. Поэтому основным случаем следует считать тот, при котором $z=$ $0, u=0, \gamma \neq 0, \varepsilon \neq 0$, т.е. $W=\widetilde{W}_{\min }, W=\widetilde{W}_{\text {max }}$ или $W=F(t)+C_{1}$, где $C_{1}=$ const, $L=L_{\min }, L=L_{\max }$ или $L=-F(t)+C_{2}$, где $C_{2}=$ const. Условия (13)-(14) можно использовать для построения областей значений оптимальных управлений. В частности, пусть

$$
\begin{aligned}
& F(t)+L_{\max }-V_{\text {min }}<F(t)-D_{\text {min }}, F(t)-D_{\text {max }}<F(t)+L_{\text {max }}-V_{\text {min }}, \\
& F(t)-D_{\text {max }}>F(t)+L_{\text {min }}-V_{\text {max }} .
\end{aligned}
$$

Область значений управлений $W(t)$ для этого случая приведена на рис. 1. Пусть оптимальное управление $W(t)$ имеет вид, представленный на рис. 2, соответственно этому управлению $W(t)$ на рис. 3 приведена область значений управления $L(t)$. Из этих двух рисунков следует:

1) если $W=W_{\min }$, то

$$
\max \left\{L_{\min }, \quad V_{\min }+W_{\min }-F(t)\right\} \leq \min \left\{L_{\max }, \quad V_{\max }+W_{\min }-F(t)\right\} ;
$$

2) если $W=W_{\max }$, то

$$
\max \left\{L_{\min }, \quad V_{\min }+W_{\max }-F(t)\right\} \leq \min \left\{L_{\max }, \quad V_{\max }+W_{\max }-F(t)\right\} ;
$$

3) если $W=F(t)-D_{\max }$, то

$$
\max \left\{L_{\min }, \quad V_{\min }-D_{\min }\right\} \leq L(t) \leq \min \left\{L_{\max }, \quad V_{\max }-D_{\max }\right\} .
$$

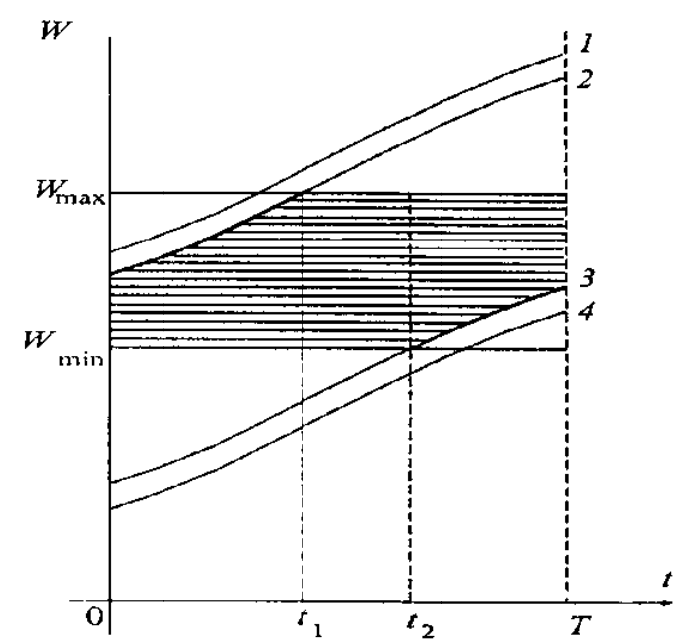

Рис. 1. Область допустимых управлений $W: 1-W=F-D_{\min } ; 2-W=F+L_{\max }-V_{\min } ; 33-W=F-D_{\max }$; $4-W=F+L_{\min }-V_{\max }$

Fig. 1. The scope of admissible controls $W: 1-W=F-D_{\min } ; 2-W=F+L_{\max }-V_{\min } ; 33-W=F-D_{\max }$; $4-W=F+L_{\min }-V_{\max }$ 


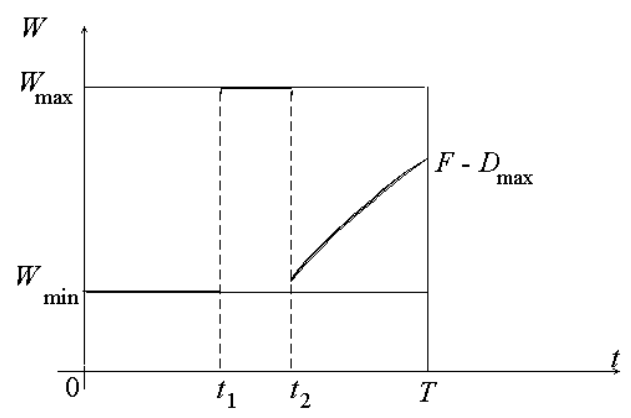

Рис. 2. График оптимального управления отбором внизу колонны

Fig. 2. Schedule optimal control selection column bottom

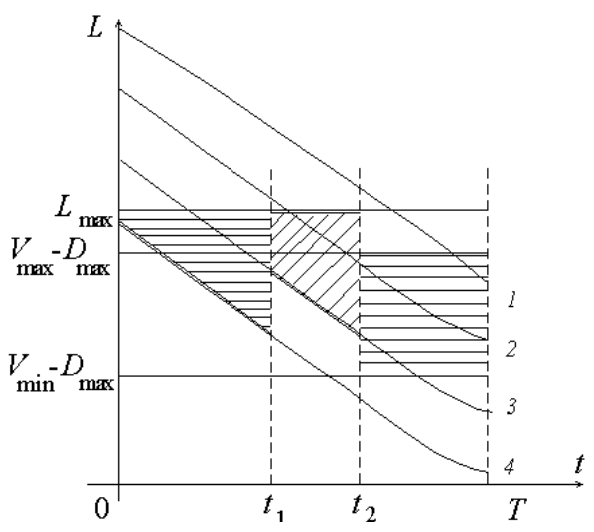

Рис. 3. Область значений управления $L$ при оптимальном управлении $W$ (см. рис. 2): $1-L=V_{\max }-F+W_{\max }$; $2-L=V_{\max }-F+W_{\min } ; 3-L=V_{\min }-F_{+} W_{\max } ; 4-L=V_{\min }-F+W_{\min }$

Fig. 3. The range of values of control $\mathrm{L}$ with optimal control W (see Fig. 2): $1-L=V_{\max }-F+W_{\max }$; $2-L=V_{\max }-F+W_{\min } ; 3-L=V_{\min }-F_{+} W_{\max } ; 4-L=V_{\min }-F+W_{\min }$

Пусть теперь график оптимального управления $W(t)$ имеет вид, приведенный на рис. 4. Область изменения управления $L(t)$ построена на рис. 5 при следующих условиях:

1) если $W=F(t)+L_{\max }-V_{\max }$, то $L=L_{\max }$;

2) если $W=W_{\max }$, то

$$
\max \left\{L_{\min }, \quad V_{\min }+W_{\max }-F(t)\right\} \leq L(t) \leq \min \left\{L_{\max }, \quad V_{\max }+W_{\max }-F(t)\right\} ;
$$

3) если $W=F(t)-D_{\max }$, то

$$
\left.\max \left\{L_{\min }, \quad V_{\min }-D_{\max }\right)\right\} \leq L(t) \leq \min \left\{L_{\max }, \quad V_{\max }-D_{\max }\right\} .
$$

Разработанный метод оптимизации применен при автоматизации промышленных ректификационных установок сернокислотного алкилирования изобутана бутиленами, получения ортоксилола и др. [1, 3, 11]. 


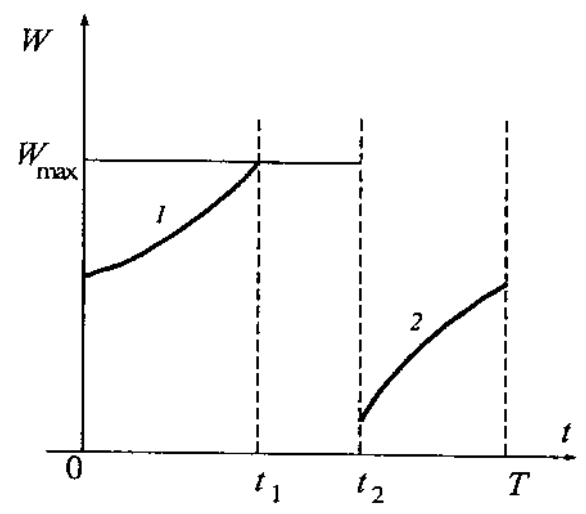

Рис. 4. График оптимального управления $W$ : $1-L=F+L_{\max }-V_{\min } ; 2-L=F-D_{\max }$

Fig. 4. Graph of optimal control $W: 1-L=F+L_{\max }-V_{\text {min }}$; $2-\mathrm{L}=\mathrm{F}-\mathrm{D}_{\max }$

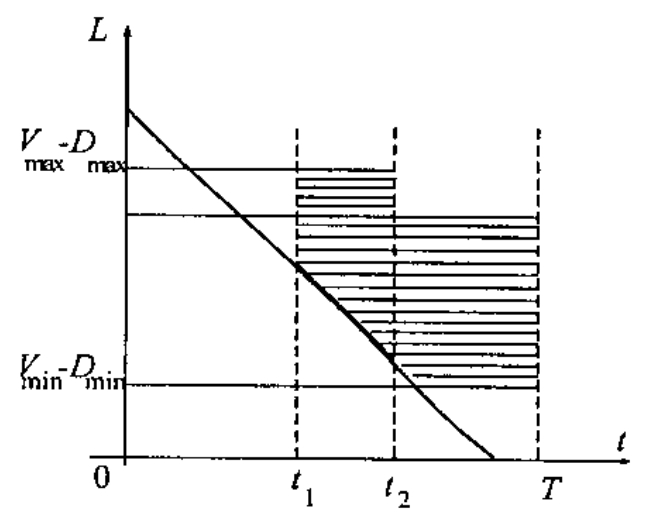

Рис. 5. Область значений управления $L=V_{\min }-F+W_{\max }$ при оптимальном управлении $W$ (см. рис. 4)

Fig. 5. Control Value Area $L=V_{\min }-F+W_{\max }$ with optimal control $W$ (see Fig. 4)

\section{Благодарность}

Исследование выполнено при финансовой поддержке РФФИ и Правительства Красноярского края в рамках научных проектов №№ 18-48-242001, 18-41-242004, 18-41-242008.

\section{Список литературы}

[1] Демиденко Н.Д. Управляемые распределенные системы. Новосибирск: Наука, 1999.393 с. [Demidenko N.D. Managed distributed systems. Novosibirsk: Nauka, 1999. 393 p. (in Russian)]

[2] Демиденко Н.Д., Кулагин В.А., Шокин Ю.И. Моделирование и вычислительные технологии распределенных систем. Новосибирск: Наука, 2012. 424 с. [Demidenko N.D., Kulagin V.A., Shokin Yu.I. Modeling and computing technologies of distributed systems. Novosibirsk: Nauka, 2012. 424 p. (in Russian)]

[3] Демиденко Н.Д., Потапов В.И., Шокин Ю.И. Моделирование и оптимизация систем с распределенными параметрами. Новосибирск: Наука, 2006. 551 с. [Demidenko N.D., Potapov V.I., Shokin Yu.I. Modeling and optimization of systems with distributed parameters. Novosibirsk: Nauka, 2006. 551 p. (in Russian)]

[4]ДемиденкоН.Д., УшатинскаяН.П.Моделирование, распределенныйконтрольиуправление проиессами ректификации. Новосибирск: Наука, 1978. 285 с. [Demidenko N.D., Ushatinskaya N.P. Modeling, distributed control and management of rectification processes. Novosibirsk: Nauka, 1978. 285 p. (in Russian)]

[5] ДемиденкоН.Д., Кулагина Л.В. Моделированиеи оптимизация техническихсистемсраспределенными параметрами. Красноярск: ИПЦКГТУ, 2006. 210 c. [Demidenko N.D., Kulagina L.V. Modeling and optimization of technical systems with distributed parameters. Krasnoyarsk: CPI KSTU, 2006. 210 p. (in Russian)]

[6] Демиденко Н.Д., Кулагина Л.В., Мельник И.Н. Анализ нестационарных режимов в системах контроля и управления распределенными процессами. Вестник СибГАУ, 2006, 2, 89-92. [Demidenko N.D., Kulagina L.V., Melnik I.N. Analysis of non-stationary modes in 
systems for monitoring and controlling distributed processes. Vestnik SibSAU, 2006, 2, 89-92. (in Russian)]

[7] Kulagina L.V. Mathematical Modeling of Control Systems for Production Processes with Distributed Parameters. Chemical and Petroleum Engineering, 2007, 43(5-6), 319-322.

[8] Демиденко Н.Д., Кулагина Л.В. Исследование систем с распределенными параметрами на базе математического моделирования. Химическое и нефтегазовое машиностроение, 2007, 3, 25-27. [Demidenko N.D., Kulagina L.V. The study of systems with distributed parameters based on mathematical modeling. Chemical and oil and gas engineering, 2007, 3, 25-27. (in Russian)]

[9] Демиденко Н.Д., Кулагина Л.В. Численное исследование систем с распределенными параметрами. Вестник КрасГАУ, 2007, 2, 103-112. [Demidenko N.D., Kulagina L.V. Numerical research of systems with distributed parameters. Vestnik KrasGAU, 2007, 2, 103-112. (in Russian)]

[10] Демиденко Н.Д. Моделирование и оптимизаџия тепломассообменных процессов в химической технологии. М.: Наука, 1991. 240 c. [Demidenko N.D. Modeling and optimization of heat and mass transfer processes in chemical technology. M.: Nauka, 1991. 240 p. (in Russian)]

[11] Демиденко Н.Д., Кулагин В.А., Шокин Ю.И., Ли Ф.-Ч. Тепломассообмен и суперкавитаиия. Новосибирск: Наука, 2015. 436 с. [Demidenko N.D., Kulagin V.A., Shokin U.I., Lee F.-C. Heat and Mass Transfer and Supercavitation, Novosibirsk: Nauka, 2015. 436 p. (in Russian)].

[12] Демиденко Н.Д., Кулагина Л.В. Численный метод исследования стационарных режимов в технологических печах. Журнал СФУ. Техника и технологии, 2014, 7(1), 55-61. [Demidenko N.D., Kulagina L.V. Numerical method for studying stationary modes in process furnaces. Journal SFU. Technics and technology, 2014, 7(1), 55-61. (in Russian)]

[13] Кашкина Л.В., Кулагин В.А., Кулагина Л.В., Стебелева О.П. Изучение эффектов кавитационного диспергирования твердофазных материалов. Энергетика в глобальном мире. Сборник тезисов, 2010. 415-416. [Kashkina L.V., Kulagin V.A., Kulagina L.V., Stebeleva OP Study of the effects of cavitation dispersion of solid-phase materials. Energetika in the global world. Collection of theses, 2010. 415-416. (in Russian)]

[14] Demidenko N.D., Kulagina L.V. Optimal Control of Thermal-Engeneering Processes in Tube Funaces. Chemical and Petroleum Engineering, 2006, 42(3-4), 128-130.

[15] Демиденко Н.Д., Кулагина Л.В. Оптимальное управление технологическими процессами в трубчатых печах. Химическое и нефтегазовое машиностроение, 2006, 3, 8-9. [Demidenko N.D., Kulagina L.V. Optimal process control in tube furnaces. Chemical and oil and gas engineering, 2006, 3, 8-9. (in Russian)]

[16] Демиденко Н.Д., Кулагина Л.В. Оптимальное управление режимами работы технологических печей в нефтеперерабатывающей промышленности. Фундаментальные исследовании, 2005, 2, 43-44. [Demidenko N.D., Kulagina L.V. Optimal control of operating modes of process furnaces in the oil refining industry. Basic research, 2005, 2, 43-44. (in Russian)]

[17] Демиденко Н.Д., Кулагина Л.В. Математическое описание процессов в технологических печах. Вестник СибГАУ, 2005, 238-239. [Demidenko N.D., Kulagina L.V. Mathematical description of processes in technological furnaces. Vestnik SibSAU, 2005, 238-239. (in Russian)]

[18] Демиденко Н.Д., Кулагина Л.В. Математическое моделирование процессов в технологических печах. Вестник СибГАУ,. 2006, 7, 91-95. [Demidenko N.D., Kulagina L.V. Mathematical modeling of processes in technological furnaces. Vestnik SibSAU, 2006, 7, 91-95. (in Russian)] 
[19] Демиденко Н.Д., Кулагина Л.В. Численное моделирование технологических режимов в трубчатых печах. Омский научный вестник, 2009, 2, 242-246. [Demidenko N.D., Kulagina L.V. Numerical modeling of technological regimes in tube furnaces. Omsky Nauchny Vestnik, 2009, 2, 242-246. (in Russian)]

[20] Кулагина Л.В., Демиденко Н.Д. Особенности моделирования процессов тепломассообмена. Компрессорная техника и пневматика, 2009, 9, 11-13. [Kulagina L.V., Demidenko N.D. Features of the modeling of heat and mass transfer processes. Compressors Technics and pneumatics, 2009, 9, 11-13. (in Russian)]

[21] Евстигнеев В.В., Кулагин В.А. Кавитация в технология очистки сточных вод. В мире научных открытий, 2010, 5(11), 87-90. [Evstigneev V.V., Kulagin V.A. Cavitation in wastewater treatment technology. In the world of scientific discoveries, 2010, 5 (11), $87-90$ (in Russian)]

[22] Дубровская О.Г., Евстигнеев В.В., Кулагин В.А. Кондиционирование сточных вод энергетических систем и комплексов. J. Sib. Fed. Univ. Eng. technol, 2011 6(4), 629-641. [Dubrovskaya OG, Evstigneev V.V., Kulagin V.A. Conditioning of waste water of power systems and complexes. J. Sib. Fed. Univ. Eng. technol., 2011 6(4), 629-641. (in Russian)]

[23] Kulagin V.A., Pyanykh T.A. Modeling of processes in supercavitation evaporator with consideration of thermodynamic effects. Chemical and Petroleum Engineering. 2014, 50(1-2), 24 29. DOI: $10.1007 / \mathrm{s} 10556-014-9848-3$.

[24] Kulagin V.A., Stebeleva O. P.,Kashkina L. V., Kulagina L. V. Preparation of carbon-containing nanostructures by cavitation technology. Chemical and Petroleum Engineering. 2011, 46, Issue 11-12, $767-773$. 